\title{
Stärken Sie als FuNRad-Mitglied die radiologische Gemeinschaft!
}

Das Forum niedergelassener Radiologen in der DRG (FuNRad) ist eine Plattform für den Erfahrungsaustausch interessierter Kolleginnen und Kollegen, die in der Niederlassung tätig sind oder sich niederlassen wollen.

Der Name FuNRad wurde mit Bedacht so gewählt, da zum einen die Freude an der Arbeit an Patientinnen beziehungsweise Patienten sowie in der Niederlassung für uns wichtig ist und wir diese erhalten und fördern wollen. Zum anderen hat das Forum dem sprichwörtlichen Anspruch als Plattform für eine effektive Vernetzung der niedergelassenen Radiologinnen und Radiologen zu dienen. Oft verengt sich nach der Niederlassung der Kontakt mit anderen Kolleginnen und Kollegen, sodass wichtige Informationen und Erfahrungen, die bereits von anderen Kolleginnen und Kollegen gemacht wurden, nicht gleichermaßen für alle verfügbar sind. Gleichzeitig sehen wir uns in der ambulanten Radiologie einer immer komplexeren Situation gegenüber, die dominiert wird von sich schnell ändernden regulatorischen Anforderungen, politischen Änderungen und einer sich drastisch ändernden Wettbewerbslandschaft innerhalb der Radiologie, bei der klassische freie radiologische Praxen beständig an Anzahl abnehmen. Um innerhalb dieser anspruchsvollen Gemengelage weiterhin optimal für die Patientenversorgung, aber auch für die Belange der eigenen Mitarbeiterinnen sowie Mitarbeiter und der Praxis sorgen zu können, sind eine effektive Kommunikation mit niedergelassenen

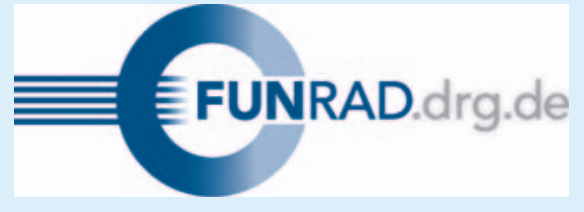

Kolleginnen und Kollegen sowie ein effektiver Erfahrungsaustausch wichtige Grundvoraussetzungen. Jedes Mitglied im FuNRad stärkt somit die Gemeinschaft und trägt für alle zu einer Verbesserung der Situation bei.

FuNRad vernetzt und vermittelt auf faire Art und Weise wichtige Informationen für radiologische Praxen. Darüber hinaus vertritt FuNRad die niedergelassenen Radiologinnen und Radiologen innerhalb der Deutschen Röntgengesellschaft, die traditionell eher universitär-klinisch geprägt ist. Aus diesem Grund möchte FuNRad dabei die häufig fehlende Facette der Bedürfnisse der niedergelassenen Radiologinnen und Radiologen innerhalb der Deutschen Röntgengesellschaft aufgreifen und vertreten. Dies hat bereits in den letzten Jahren auf dem Röntgenkongress zur Einführung einer dezidierten Vortragsserie für niedergelassene Radiologinnen und Radiologen geführt, die sehr gut von allen Teilnehmenden des Kongresses angenommen wurde auch von klinisch tätigen Kolleginnen und Kollegen. Wir sehen uns mit diesem Erfolg bestätigt, diesen eingeschlagenen Kurs weiterzuverfolgen. Dies kann umso besser erfolgen, je spezifischer die Wünsche der niedergelassenen Kolleginnen und Kollegen an uns herangetragen werden. Einen besonderen Fokus wollen wir dabei in den nächsten Jahren auf den Zeitraum vor der Niederlassung legen und dabei den interessierten Kolleginnen und Kollegen einen realistischeren Einblick in die Arbeit als niedergelassene Radiologinnen/niedergelassene Radiologen geben. Flankierend sollen dabei Informationen zum technischen Ablauf eines Einstiegs und die damit auch einhergehenden finanziellen Aspekte gegeben werden.

Wir sehen uns explizit nicht als berufspolitische Vereinigung. Dieser Themenbereich fällt dem Bundesverband Deutscher Radiologen zu, der jedoch mit FuNRad eng kooperiert. Aus diesem Grund ist auch der Vorstand des Bundesverbands der Deutschen Radiologen kooptiertes Mitglied im FuNRadVorstand.

Mitgliedsanträge für FuNRad können alle interessierten Mitglieder der DRG und/oder des BDR bei der Geschäftsstelle über die DRG stellen. Alle weiteren Informationen erhalten Sie unter www.funrad.drg.de.

Die Mitgliedschaft im FuNRad ist kostenfrei. Wir freuen uns auf Sie!

Ihr

Prof. Dr. med. Henrik Michaely, Vorstandsvorsitzender des Forums niedergelassener Radiologen 